\title{
Multilevel topological analysis in application to design of coordination networks
}

Eugeny V. Alexandrov ${ }^{1}$, Alexander P. Shevchenko ${ }^{1}$, Vladislav A. Blatov ${ }^{1}$, Davide M. Proserpio ${ }^{2}$ ${ }^{1}$ Samara University, Samara, RUSSIAN FEDERATION, ${ }^{2}$ Dipartimento di Chimica, Università degli Studi di Milano, Milan, Italy E-mail: aleksandrov_ev1@mail.ru

Topological methods of analysis of coordination networks have become an integral part of design of coordination polymers, MOFs, and zeolites. It helps to chemists to understand the novelty of findings, to reveal relations between different structures, to propose new design concepts, and to communicate effectively with each other. However, this is not the limit of the methods. Description of coordination environment of structural units (metal atoms, ligands, SBUs), network topology (topological type), and topology of entanglement (entanglement pattern) intrinsically leads to finding relations between the topological parameters. Further, the correlations can be grouped (systematized) in a hierarchical manner to produce a knowledge database for design and prediction of new materials (their structure and properties) [1, 2]. Thus, new structural characteristics, the so-called descriptors, have to be invented for prediction of particular structural features.

For example, we have proposed to use topological type of Hopf ring net for analysis of topology entanglements in coordination networks. Now we put forward Extended ring net (ERN), which incorporates information about topology of entangled nets and topology of entanglements. ERN is able to distinguish patterns of entanglements up to isomorphism and polymorphism, that allowed us to describe new type of isomerism of coordination polymers, entanglement isomerism.

MOF structures are usually considered as a result of assembly of secondary building units in an isoreticular manner. Usually SBU is considered as a topologically dense OD polynuclear complex group, which coordination figure is predetermined by orientation of points of extension (functional groups of ligands connected by linkers). However, many practically important MOFs contain infinite SBUs; e.g. rod-shaped $1 \mathrm{D}\{\mathrm{AlOH}(\mathrm{CO} 2) 2\}$ groups are connected by benzene linkers in parallel fashion into framework MIL-53. We have produced a comprehensive systematics of infinite SBUs and ways for their connection with new algorithms of rod-MOFs description as 2D net of rods connections.

Selection of correct structural groups is also important for design of self-catenated networks. We proposed a universal algorithm for selection of all reasonable subnets and determining the structure driving ones.

To describe the geometry and topology of voids and channels in porous structures we combined topological methods and Voronoi partitioning. The improved Voronoi partitioning provides much more characteristics of porous structures, e.g. periodicity, topology and crystallographic orientation of channels, minimal cages of framework, open sights of metal atoms etc. These descriptors give us an opportunity to define metal-organic nanotube as a 1-periodic tiling of face-shared 3D tiles. To enhance the usability of the results of topological analysis we collected values of descriptors into a knowledge database [2]. The database was applied in finding relations between topological parameters for heterometallic coordination polymers based on $\{\mathrm{Cu}(\mathrm{Me} 2 \mathrm{mal}) 2\} \mathrm{SBU}$ (H2Me2mal = dimethylmalonic acid) [3].

The authors thank the Russian government (grant No. 14.B25.31.0005), Russian Science Foundation (grant No. 16-1310158), and Russian Ministry of Science for finantinal support. E.V.A. is gratefull to Russian Foundation for Basic Research (grant No. 16-37-00147).

[1] Alexandrov, E.V. et al. (2015) Chem. Rev. 115, 12286-12319. rn[2] Alexandrov, E.V. et al. (2015) CrystEngComm., 17, 2913-2924. rn[3] Gogoleva, N. V. et al. (2016) Eur. J. Inorg. Chem., doi: 10.1002/ejic.201601047.

Keywords: Topological analysis, coordination networks, crystal design 